\title{
EXPLICIT TRANSITIVE AUTOMORPHISMS OF THE CLOSED UNIT SQUARE
}

\author{
XIAO-QUAN XU \\ (Communicated by Dennis Burke)
}

Abstract. We construct transitive automorphisms of the closed unit square.

In [2], Oxtoby has made the following statement: "An explicit example of a transitive automorphism of the plane was given by Besicovitch, but it is not easy to exhibit one for the closed unit square $\cdots$. The existence of such transformations was first established by the category method". In this note, based on Besicovitch's transitive transformations of the plane, we will construct some transitive automorphisms of the closed unit square $I^{2}$.

Let $S$ be any Besicovitch transitive transformation of the plane [1],

$$
S\left(\mathrm{re}^{i \varphi}\right)=\mathrm{re}^{f(\varphi)} e^{i(\varphi+2 \pi \theta)}
$$

where $f$ is any continuous function with period $2 \pi$ satisfying the two following conditions:

(1) $\int_{0}^{2 \pi} f(\varphi) d \varphi=0$

(2) For every $\delta \in R$ and every positive integer $n$, the function $F(\varphi, \delta, n)=\sum_{j=0}^{n-1} f(\varphi+j \delta)$ is constant in no interval of values of $\varphi$.

$\theta=\theta(f)$ is some irrational number in $(0,1)$ [1]

Let $D=\left\{(x, y) \in R^{2}: x^{2}+y^{2} \leq 1\right\}$. Define $H: D \rightarrow D$,

$$
H\left(\mathrm{re}^{i \varphi}\right)=\frac{\mathrm{re}^{f(\varphi)}}{1-r+\mathrm{re}^{f(\varphi)}} e^{i(\varphi+2 \pi \theta)}, \quad 0 \leq r \leq 1, \quad 0 \leq \varphi<2 \pi .
$$

For every homeomorphism $\Phi: I^{2} \rightarrow D$, let $T=\Phi^{-1} \circ H \circ \Phi: I^{2} \rightarrow I^{2}$. Then $T$ is a transitive automorphism of the closed unit square.

In fact, $G$ : Int $D \rightarrow R^{2}$,

$$
G\left(\mathrm{re}^{i \varphi}\right)=\frac{r}{1-r} e^{i \varphi}, \quad 0 \leq r<1, \quad 0 \leq \varphi<2 \pi
$$

Received by the editors June 23, 1989.

1980 Mathematics Subject Classification (1985 Revision). Primary 54H20, 34C35.

Research supported by the National Science Fund of China. 
is a homeomorphism; therefore, $G^{-1} \circ S \circ G$ : Int $D \rightarrow$ Int $D$ is a transitive transformation of Int $D$. By calculation we know

$$
G^{-1} \circ S \circ G\left(\mathrm{re}^{i \varphi}\right)=\frac{\mathrm{re}^{f(\varphi)}}{1-r+\mathrm{re}^{f(\varphi)}} e^{i(\varphi+2 \pi \theta)} .
$$

So $H: D \rightarrow D$ is a transitive transformation of $D$. Whence $T=\Phi^{-1} \circ H \circ \Phi$ : $I^{2} \rightarrow I^{2}$ is a transitive transformation of $I^{2}$.

\section{REFERENCES}

1. A. S. Besicovitch, A problem on topological transformation of the plane, Fund. Math. 28 (1937), 61-65.

2. J. C. Oxtoby, Measure and category, Springer-Verlag, New York, 1971, 65-73.

Department of Computer Science, Jiangxi Industrial University, Nanchang, JiangXi, PEOPLE'S REPUBlic OF CHINA 